\title{
Parameter Estimation and Verification of Unmanned Air Cushion Vehicle (UACV) System
}

\author{
Mohd Zamzuri Ab Rashid ${ }^{1, *}$, Hairol Nizam Mohd Shah ${ }^{1}$, Md Nazri Othman ${ }^{1}$, Esmail ali Ali \\ Alandoli ${ }^{1}$, Mohd Shahrieel Mohd Aras ${ }^{1}$, Marizan Sulaiman ${ }^{1}$, Mohd Faruq Abdul Latif ${ }^{2}$ \\ ${ }^{1}$ Faculty of Electrical Engineering, Universiti Teknikal Malaysia Melaka, Hang Tuah Jaya, 76100 \\ Durian Tunggal, Melaka, Malaysia. \\ ${ }^{2}$ Faculty of Engineering Technology, Universiti Teknikal Malaysia Melaka, Hang Tuah Jaya, 76100 \\ Durian Tunggal, Melaka, Malaysia.
}

\begin{abstract}
This project is mainly about the dynamic modelling and parameter estimation of Unmanned Air Cushion Vehicle (UACV). The purpose of developing mathematical model of the Unmanned Air Cushion Vehicle (UACV) is due to its under actuated nonlinearities where it has less input compared to the output required. This system able to maneuver over land, water and other surfaces either at certain speed or maintain at a stationary position. In order to model the UACV, the system is set to have two propellers which are responsible to lift the vehicle by forcing high pressure air under the system. The air inflates the "skirt" under the vehicle, causing it to rise above the surface while another two propellers are used to steer the UACV forward. UACV system can be considered as under actuated since it possess fewer controller inputs that its degree of freedom. The system's motions are defined by the six degrees of freedom which are; heaved, sway and surge. Another three components are rotational motions which can be elaborated as roll, pitch and yaw. The problem related to $\mathrm{UACV}$ is normally related to obtaining accurate parameters of the system to be included into the mathematical model of the system. This is due to the body inertia of the system during the static and moving condition. Besides, the air that flows into the UACV skirt to create the cushion causes imbalance and will affect the system stability and controllability. In this research, UACV need to be mathematically modelled using Euler-Lagrange method. Then, parameters of the system can be obtained through direct calculation and Solidworks software. The parameters acquired are compared and verified using simulation and experimental studies.
\end{abstract}

\section{Introduction}

This paper presents parameter estimation and verification of Unmanned Air Cushion Vehicle System (UACV). UACV is a type of Air Cushion Vehicle (ACV) where ACV is driven by human while UACV maneuver autonomously. UACV is a system that capable to move forward and reverse, turn left and right, and able to turn around 360 degrees by utilizing air

\footnotetext{
Corresponding author: zamzuri@utem.edu.my
} 
cushion that is generated underneath. It also can lift on underling surface and it is considered as advantages compared to other vehicles such as cars and ships. This UACV can be lifted by air flow through the air chamber between the deck, skirt and ground surface using a motor's propeller while another two propellers are used to provide air thrust which to control the movement of the UACV. In order to determined the parameters of the UACV system, a UACV has been designed and drafted using the SOLIDWORKS software. Then, the parameters of the UACV such as mass, mass moment of inertia, centre of gravity are generated based on the materials assigned on the system design. The parameters acquired then are inserted into the mathematical model developed earlier. The system model is simulated and compared with the experimental platform in order to verify the parameters acquired. The experimental setup is fabricated by utilizing materials like Perspex, aluminum, steel and plastic. The IMU sensor is used in this project to detect the movement of these UACV in surge, heave and also yaw. The objectives of this project are summarized as follows: (i) To design and acquire parameters via SolidWork software and develop of the laboratory scale of UACV, (ii) To verify the parameters of the UACV system in surge, heave and yaw by simulation and experimental studies.

\section{Motivations}

UACV system is a nonlinear system which has six degrees of freedom and quite challenging to control its movement. The challenge poses by this system has attracted various researchers to conduct and explore several fields such as system design, controller design, test the performance either using simulation or experimental studies. In this literature reviews, several past researches done by expertise have been thoroughly analyzed. In the paper by [1], the author had designed a lightweight UACV that has mass below $1.5 \mathrm{~kg}$ and consists of the motor, batteries and other accessories. It was designed in a rectangular shape to ensure it has sufficient mounting points to allocate additional electronic and hardware and to maximize the surface area. The body of UACV was made by polystyrene. Two motors that were selected and used to lift the UACV are Johnson 400 that motors were secured on the top deck by hot glue and using 4 inches propeller. Next, in [2], the original air cushion vehicles with four cockpit controls were reduced to two, where turning rate and velocity were independently commanded. It was designed to cater its weight payload over surface of water. A throttle of this ACV was used to accelerate the system, while the steering wheel was to turn the ACV as resulting control system. In order to improve good handling, steering wheel deflection yield a turn rate proportional to the wheel angle. The author in [3] developed a high performance and compact a small size one seated ACV vehicle. The design combined a concept of bag skirt, peripheral jet and perforation was proposed for suitability in urban context.

However, in another paper done by [4] required novel sensory system for UACV where this task differed from conventional approach. This UACV was designed with the implementation of optic flow-based autopilot, a tiny magnetic compass and a micro-gyrometer. The two minimalist eyes were mounted sideway opposite to each other, and give the lateral Optic Flow (OF) to be measured. The paper in author [5] using the RCX controller that wired on the UACV and it will receive the signal from the computer using a USB tower. The program was transfered by the tower to the controller to operate the hovercraft system. The main purpose of the program was to have the light sensors recognize specific colors and to turn the fans ON and OFF so the UACV can move about. This author had two different programs that were written for their project. The first program was written to have the sensors followed a white path that was bordered in by two black strips on either side, which also guided it and for the second program was written to have the sensors to follow a single black strip of tape that would guide the UACV along its path. 
Besides that in [6], based on the adaptive dynamic inversion control scheme, a neuralnetwork conjunction with a line-of-sight guidance law was adopted in this work for the under actuated UACV followed a prescribed path. Next, the author wrote this paper in [7] to develop a new algorithm that account for bounded sensor noise and bounded model uncertainty by the dynamic scheduling of multiple receding horizon control of the systems. Other than that, from the author from [8] was written about a dynamic second-order sliding mode control of the hovercraft vessel. This is the combination of the second-order sliding mode and the differential flatness property controller design technique. This kind of control proposed for the specification of a robust dynamic feedback multivariable controller. It was used to track earth coordinate position of a hovercraft vessel model.

From [9], the author had designed wing of the UACV. When UACV had reached a sufficient horizontal speed, it will lift the vehicle just off the surface as by the ground effect. Although the air cushion vehicles had same weight to helicopter, the UACV consumed less power than the helicopter. The system also can attain higher speed compare to mobile vehicle or ships. The UACV contained four control parameters of $\mathrm{x}, \mathrm{y}$ and $\mathrm{z}$-coordinate and orientation angle, much like standard as automobile. It was controlled with horizontal and vertical thrust and motion of rudders control the orientation. This system was open loop. It could be developed to be a closed loop by develop a feedback module in the form of monitoring of various operation of hovercraft. As to track the vehicle movements, it used a GPS guidance system.

\section{System design}

From the previous studies inside section II shown that most of the researches focused on the design and controller to control the movement either ACV or UACV systems. The system that is designed in this project consists of one propeller driven by a brushless motor to provide a lift thrust while another two propellers to provide a thrust for the system to move forward or during turning movement. Figure 1 shows the degree of freedom associated with this UACV system while Figure 2(a) - 2(d) show the isometric view, top view, side view and back view of the UACV system that have been drafted using the SOLIDWORKS software. Another figure, Figure 3(a) - 3(d) shows the experimental setup used in order to obtain more accurate information related with UACV system.

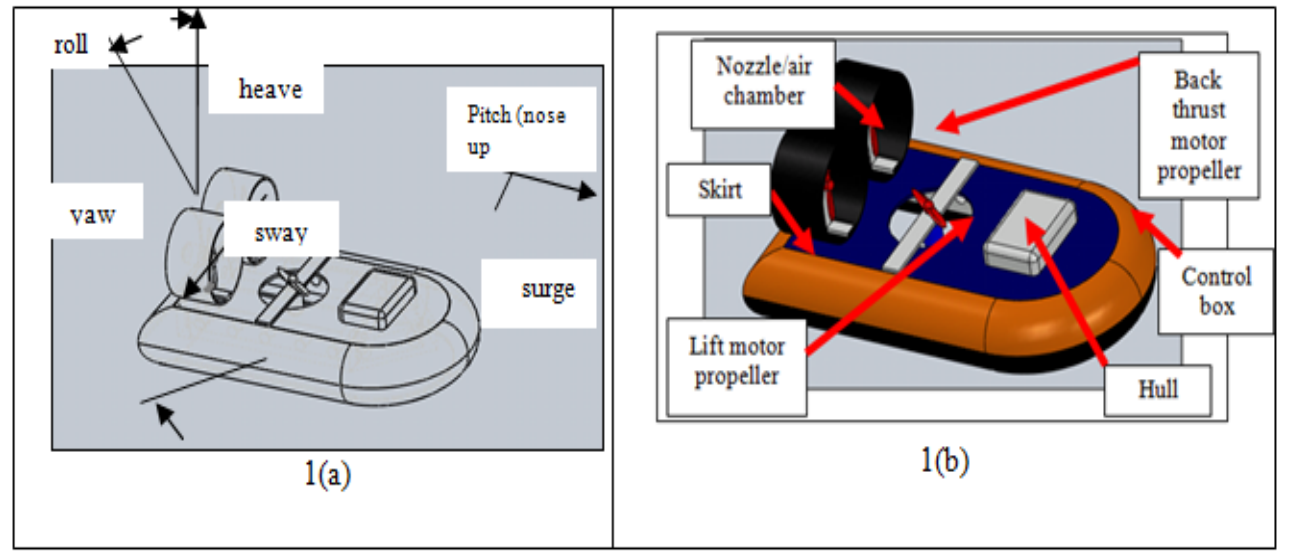

Fig. 1. (a) UACV degree of freedom, (b) UACV design 


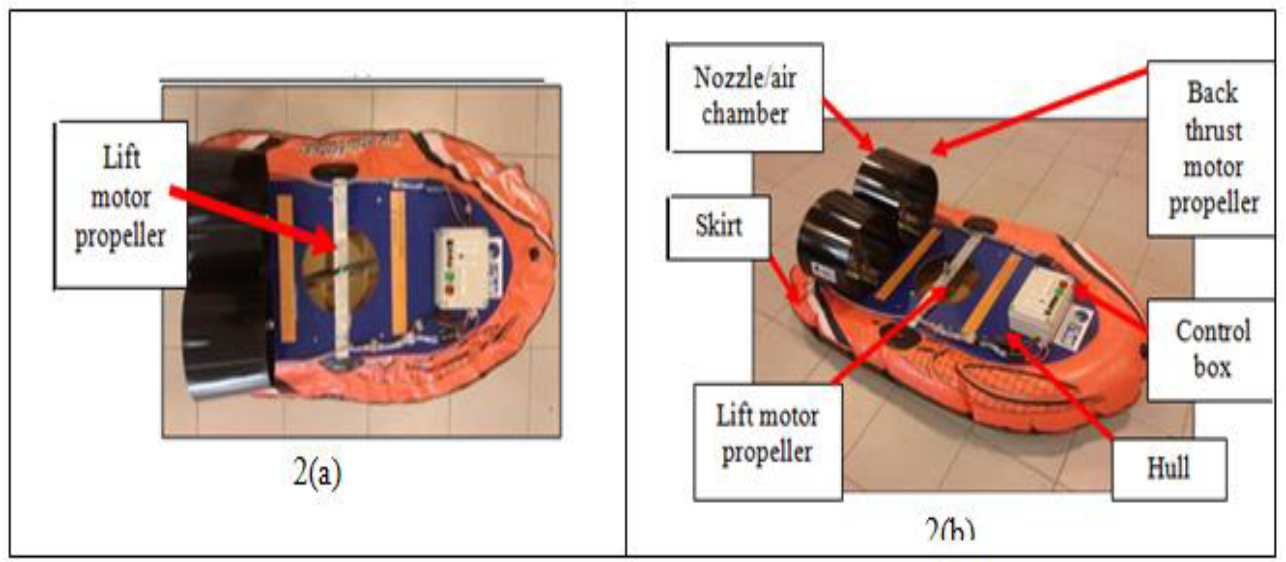

Fig. 2. (a) Top View of UACV Setup, (b) Isometric View of UACV

\section{Results and discussion}

Analysis have been done on the SOLIDWORKS design and the parameters of the UACV system have been obtained. Table 1 shows the parameters that have been acquired from the SOLIDWORKS sofware and moment of inertia (I) $=19.823 \mathrm{kgm}^{2}$

Table 1. Parameters of UACV

\begin{tabular}{|c|c|c|}
\hline Parameters & Value & Unit \\
\hline Mass, $\mathrm{m}$ & 10.57 & $\mathrm{~kg}$ \\
\hline Volume & 0.032 & $\mathrm{~m}^{3}$ \\
\hline Surface area & 4.429 & $\mathrm{~m}^{2}$ \\
\hline Center of mass & $\mathrm{X}=0.500$ & $\mathrm{~m}$ \\
\cline { 2 - 3 } & $\mathrm{Y}=1.000$ & $\mathrm{~m}$ \\
\cline { 2 - 3 } & $\mathrm{Z}=0.400$ & $\mathrm{~m}$ \\
\hline \multirow{3}{*}{ Moment of inertia } & $\mathrm{I}_{\mathrm{xx}}=$ & $\mathrm{kgm}^{2}$ \\
\cline { 2 - 3 } & $\mathrm{I}_{\mathrm{yy}}=5.487$ & $\mathrm{kgm}^{3}$ \\
\cline { 2 - 3 } & $\mathrm{I}_{\mathrm{zz}}=$ & $\mathrm{kgm}^{4}$ \\
\hline
\end{tabular}

The parameters odtained tnen are inciuded into tne matnematicaı model done by [13]. The state space is shown in Equation (3):

$$
\dot{x}=A x+B u, \text { and } y=C x+D u
$$

where:

$$
A=\left[\begin{array}{cccccc}
0 & 0 & 0 & 0.988 & 0 & 0 \\
0 & 0 & 0 & 0 & 0.154 & 0 \\
0 & 0 & 0 & 0 & 0 & 1 \\
-1 & 0 & 0 & 0 & 0 & 0 \\
0 & -1 & 0 & 0 & 0 & 0 \\
0 & 0 & -1 & 0 & 0 & 0
\end{array}\right], B=\left[\begin{array}{ccc}
0 & 0 & 0 \\
0 & 0 & 0 \\
0 & 0 & 0 \\
0.094 & 0 & 0 \\
0 & 0.094 & 0 \\
0 & 0 & -19.823
\end{array}\right]
$$

$x=[x, y, z, r, p, \varphi]^{T} u=\left[\begin{array}{lll}\tau_{u} & 0 & \tau_{r}\end{array}\right]$ 
All the state space obtained has been included into the Matlab/SIMULINK and in order to prove simulation studies, a few experimental studies have been carried out and the outcomes of these experiments are elucidated in Figure 3 to Figure 4. The movement in straight line has a little bit vibration during the initial start but UACV moves steadily after certain period of time as shown in Figure 3(c).

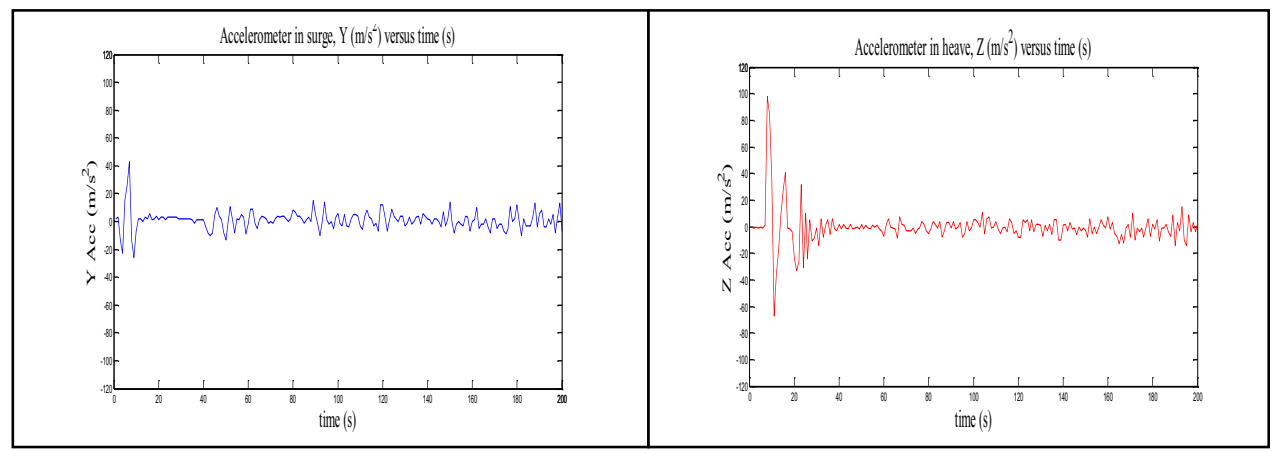

Fig. 3. (a) Acceleration in surge

Fig. 3. (b) vertical lift (heave)

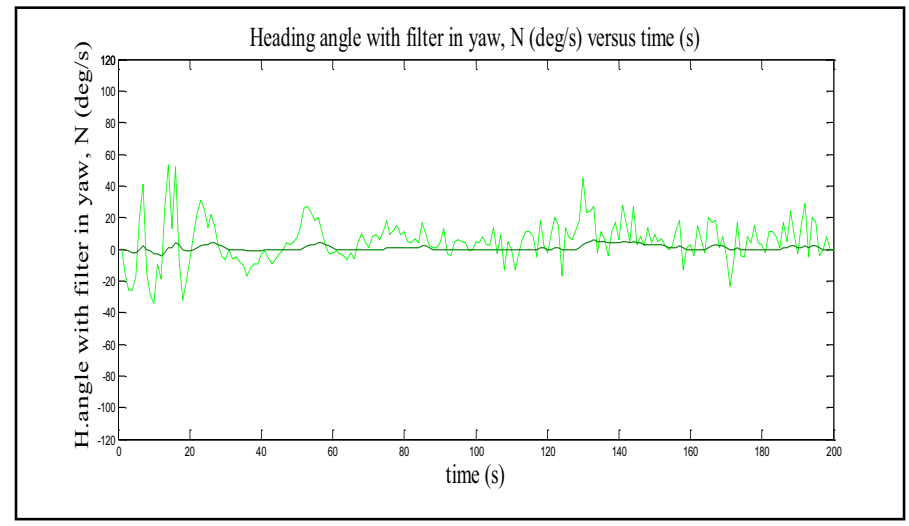

Fig. 3. (c). heading angle (yaw) during experimental straight motion 


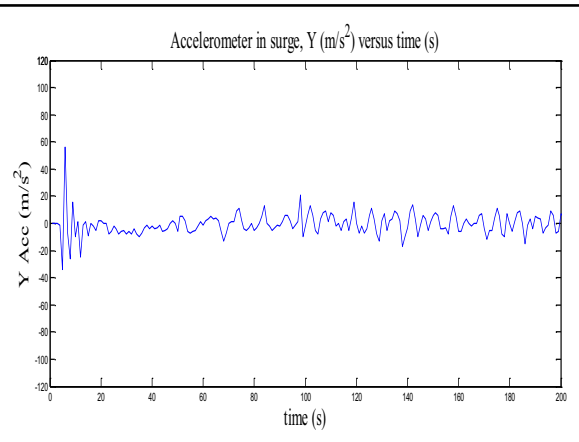

4(a)

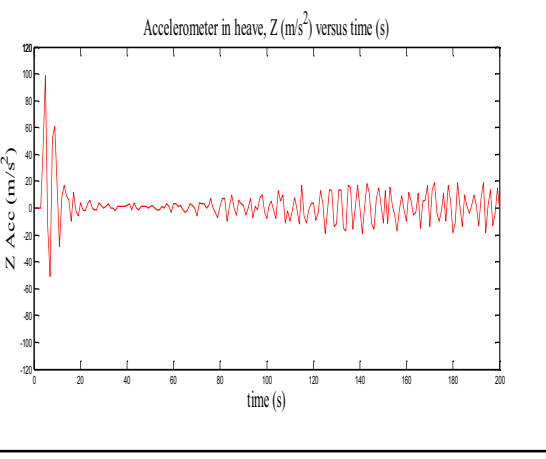

4(b)

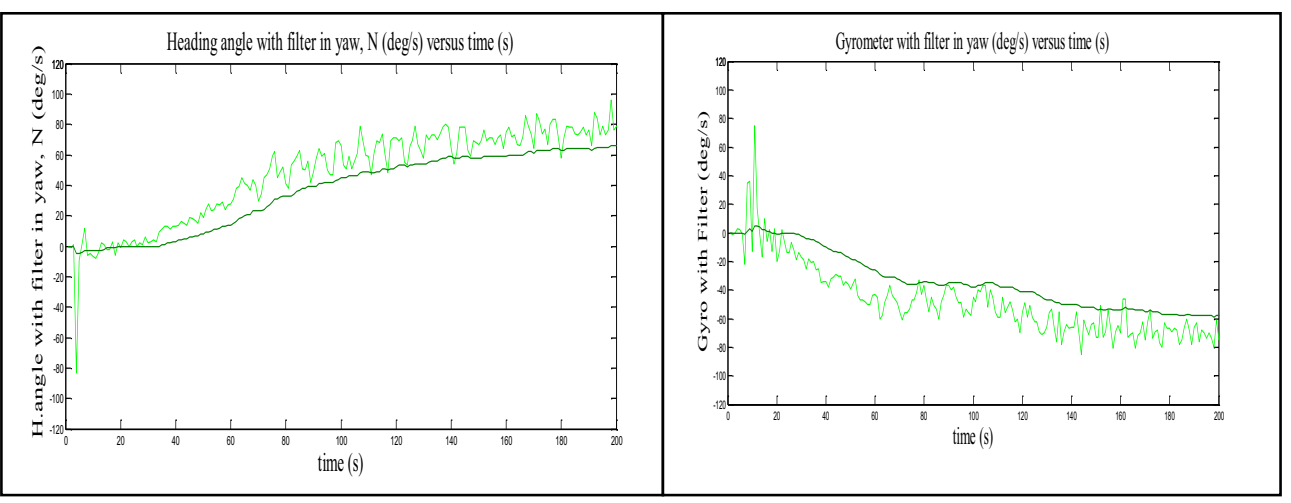

4(c)

4(d)

Fig. 4. (a) Acceleration in surge, (b) vertical lift (heave), (c) heading angle (yaw) during right turn , (d) heading angle (yaw) during left turn

Figure 4(a) to Figure 4(c) show the movement of the UACV when it makes a right turn while 4(d) shows the movement of UACV when turn.

\section{Conclusion}

In conclusion, the UACV system has been developed and its parameters have been included into the mathematical model and simulation studies have been carried out. Then, the experimental studies have been conducted and the data obtained from the experimental studies are consistent with the simulation result.

The author want to express appreciation to Unmanned and Autonomous Research Group (UAIR), Centre of Excellence in Robotics and Industrial Automation (CERIA), Universiti Teknikal Malaysia Melaka (UTeM) and Ministry of Higher Education, Malaysia for sponsoring this project under RAGS grant no RAGS /1/2014/TK03/FKE/B00057.

\section{References}

1. M.Conyers, A.Walker, B.Warwick, Mechatronics Seng, 466 University of Victoria, Report the Second Phase of the Spring (2010) 
2. R. Gran, G. Carpenter, R.W. Klein, Computer Aided design of a Control System for a Hovercraft. Decision and Control, 1982 21st IEEE Conference on. 1347-1353 (1982)

3. S.M. Hein, H.C. Liaw, International Conference on Advanced Intelligent Mechatronics, $1516(2013)$

4. F.L. Roubieu, J. Serres, N. Franceschini, F. Ruffier, S. Viollet, IEEE International Conference, 1311 (2012) 